Title:

\title{
Generation and clinical application of gene modified autologous epidermal sheets in Netherton syndrome - lessons learned from a phase 1 trial
}

Authors: Wei-Li Di ${ }^{1^{*}}$, Su M Lwin ${ }^{2+}$, Anastasia Petrova ${ }^{1+}$, Catina Bernadis ${ }^{3}$, Farhatullah Syed ${ }^{1}$, Farzin Farzaneh ${ }^{4}$, Dale Moulding ${ }^{5}$, Anna E Martinez ${ }^{6}$, Neil J Sebire ${ }^{7}$, Dyanne Rampling ${ }^{7}$, Alex Virasami ${ }^{7}$, Mozheh Zamiri ${ }^{8}$, Wei Wang ${ }^{9,10}$, Havinder Hara ${ }^{1}$, Tendai Kadiyirire $^{2}$, Alya AbdulWahab $^{2}$, Magdalena Martinez-Queipo ${ }^{2}$, John I Harper ${ }^{1}$, John A McGrath ${ }^{2}$, Adrian J Thrasher $^{1}$, Jemima E Mellerio ${ }^{2}$ and Waseem Qasim ${ }^{1}$

1. Infection, Immunity and Inflammation Programme, UCL GOS Institute of Child Health, 30 Guilford Street, London WC1N 1EH, UK

2. St John's Institute of Dermatology, King's College London, Guy's Hospital, Great Maze Pond, London SE1 9RT, UK

3. Plastic Surgery Unit, Guy's and St Thomas' NHS Foundation Trust, Westminster Bridge Rd, Lambeth, London SE1 7EH, UK

4. Cell \& Gene Therapy - King's (CGT-K), School of Cancer and Pharmaceutical Sciences, King's College London, 123 Coldharbour Lane, London SE5 9NU, UK

5. Light Microscopy Core Facility, UCL GOS Institute of Child Health, 30 Guilford Street, London WC1N 1EH, UK

6. Dermatology Department, Great Ormond Street Hospital NHS Foundation Trust, Great Ormaond Street, London WC1N 3JH, UK

7. Histopathology Department, Great Ormond Street Hospital for Children NHS Trust, Great Ormond Street, London WC1N 3JH, UK

8. School of Life Sciences, University of Dundee, Dow Street, Dundee, DD1 5EH, Scotland, UK

9. Department of Translational Oncology, German Cancer Research Center, National Center for Tumor Diseases, Heidelberg, Im Neuenheimer Feld 581, 69120, Heidelberg, Germany

10. GeneWerk GmbH, Im Neuenheimer Feld 582, 69120, Heidelberg, Germany 
* Correspondence should be addressed to

Dr Wei-Li Di

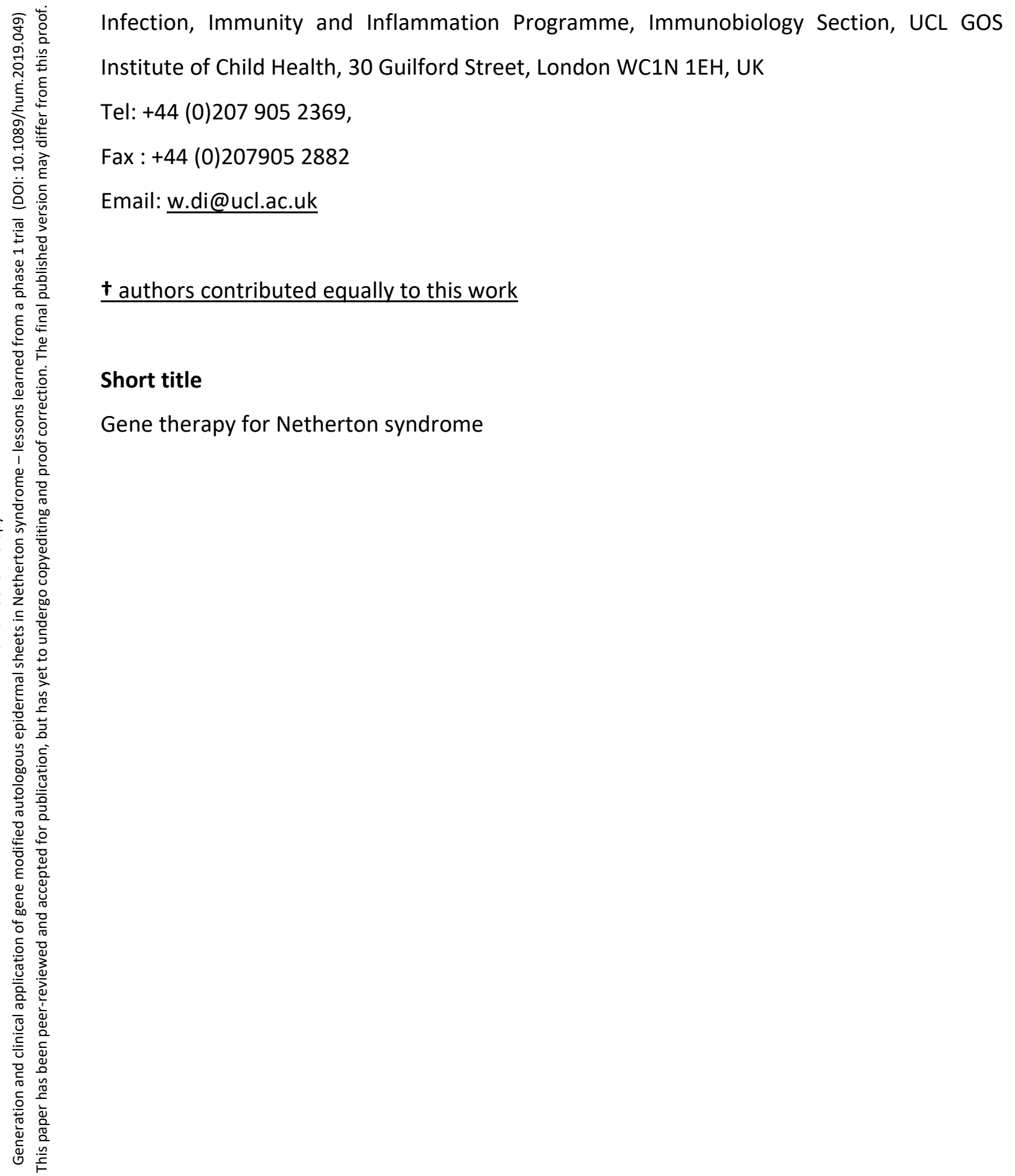




\section{Abstract}

Netherton Syndrome (NS) is a rare autosomal recessive skin disorder caused by mutations in SPINK5. It is a debilitating condition with notable mortality in the early years of life. There is no curative treatment. We undertook a non-randomized, open-label, feasibility and safety study using autologous keratinocytes transduced with a lentiviral vector encoding SPINK5 under the control of the human involucrin promoter. Six NS subjects were recruited and gene-modified epithelial sheets were successfully generated in three out of five subjects. The sheets exhibited expression of correctly sized LEKTI protein after modification. One subject was grafted with a $20 \mathrm{~cm}^{2}$ gene-modified graft on the left anterior thigh without any adverse complications and was monitored by serial sampling for 12 months. Recovery within the graft area were compared against an area outside by morphology, pro-viral copy number and expression of the SPINK5 encoded protein, LEKTI, and its down-stream target kallikrein 5, which exhibited transient functional correction.

The study confirmed the feasibility of generating lentiviral gene-modified epidermal sheets for inherited skin diseases such as NS, but sustained LEKTI expression is likely to require the identification, targeting and engraftment of long-lived keratinocyte stem cell populations for durable therapeutic effects. Important learning points for the application of gene modified epidermal sheets are discussed. 


\section{Introduction}

Netherton syndrome (NS) is a rare autosomal recessive skin disorder caused by mutations in the gene SPINK5 ${ }^{1}$. It is a debilitating condition characterized by ichthyosiform erythroderma, atopic diathesis and defective skin barrier at or soon after birth ${ }^{2}$. The prognosis of NS is poor with notable mortality in the early years of life due to life-threatening complications such as bronchopneumonia, sepsis and hypernatraemic dehydration secondary to severe water loss through the defective skin barrier. The causative gene SPINK5 encodes the protein lympho-epithelial Kazal-type-related inhibitor $(\mathrm{LEKTI})^{3}$. It is a serine protease inhibitor expressed in the uppermost compartments of the epidermis, and plays a critical role in the regulation of two serine proteases kallikrein 5 and 7 (KLK) which hydrolyze the extracellular corneodesmosomes in the skin ${ }^{4,5}$. In NS patients, mutations in SPINK5 cause truncated protein expression, resulting in loss of activity of LEKTI 6, 7. As a consequence, hyper-activated kallikreins excessively degrade corneodesmosomes and cleave pro-elastase 2, leading to a defective skin barrier ${ }^{\text {5, 8-11. }}$.

Current treatments for NS are largely symptom based, relying on the use of emollients, corticosteroids, and antibacterials, but these treatments have limited benefit. Gene therapy offers the prospect of disease amelioration of genetic skin disorders. Gene-based correction of junctional epidermolysis bullosa (JEB) was first reported over a decade ago following ex-vivo gamma-retroviral transduction of epidermal stem cells and grafting of corrected epidermal sheets ${ }^{12}$. That study provided important proof-of-principle evidence that correction of skin stem cells can afford effective therapy of genetic skin conditions. A similar approach was recently reported for treatment of a subject with JEB with complete epidermal loss across $80 \%$ of body surface area ${ }^{13}$ and there are ongoing skin sheet gene therapy trials for recessive dystrophic epidermolysis bullosa (RDEB) in USA ${ }^{14}$ and Austria (ClinicalTrials.gov: http://clinicaltrials.gov/number; under identifier number NCT02984085 and NCT03490331).

We previously modelled gene therapy for the correction of NS using ex-vivo lentiviral modified epidermal sheets in human:murine chimeras ${ }^{15}$. We developed a third-generation self-inactivating lentiviral vector encoding a codon optimized cDNA for SPINK5 under the control of a 572bp enhancer derived from human involucrin promoter ${ }^{16}$. Reversal of abnormal NS features and restoration of normal human skin architecture in gene 
corrected grafts with compartment specific reconstitution of LEKTI expression was confirmed in humanized chimeras ${ }^{15,16}$. We now report the application of a Good Manufacturing Practice (GMP) compliant process for generating gene modified epithelial sheets ${ }^{17}$ in a feasibility and safety study for patients with NS, and report 12 month followup results from the first grafted patient.

\section{Materials and Methods}

\section{Study approval}

The trial was approved by the Gene Therapy Advisory Committee (GTAC, Ref No. GTAC188), and the Medicines \& Healthcare Products Regulatory Agency (MHRA, Ref No. EUDRACT 2011-003212-22). It was registered with ClinicalTrials.gov (http://clinicaltrials.gov/number) under identifier number NCT01545323. All patients recruited in the study provided written informed consent.

\section{Clinical trial protocol}

Detailed information about the recruitment criteria of subjects, consent forms, the preparation of GMP compliant lentiviral vector stocks, the production and release of investigation medicinal products (IMPs), grafting of gene modified epithelial sheets and schedule of clinical monitoring were published previously ${ }^{17}$.

\section{Patient recruitment, consent and skin procurement}

Patients aged 16 years or older with a clinical diagnosis of NS were identified from Guy's and St Thomas' Hospitals and referrals from NHS Ayrshire and Arran University Hospital Crosshouse, Scotland. After confirmation of biallelic SPINK5 mutations, patients were invited to participate in the clinical trial. Following informed consent, patients with NS were screened against the eligibility criteria as previously published 17 . Those eligible were recruited for the study and a $6 \mathrm{~mm}$ punch biopsy was obtained from an area of intact, uninflamed skin under local anesthesia (1\% xylocaine). The skin procurement was then sent to Gene Therapy laboratory for manufacture of gene modified epithelial sheet. Grafting took place in the out-patient theatres at St Thomas' Hospital and follow-up visits took place in the dermatology clinics at Guy's Hospital. 


\section{Mutation detections in SPINK5}

Mutations in SPINK5 were confirmed by direct Sanger sequence analysis by North East Thames Regional Genetics Laboratory, Great Ormond Street Hospital NHS Foundation Trust.

\section{Keratinocyte culture, transduction and epithelial sheet culture for the generation of IMP}

The production of GMP compliant IMP was carried out at the Gene Therapy Laboratory, Great Ormond Street Hospital (GOSH) NHS Foundation Trust, a GMP compliant facility approved by Medicines \& Healthcare products Regulatory Agency (MHRA) in the UK (MIA(IMP) 17328).

Primary keratinocytes were isolated from a $6 \mathrm{~mm}$ skin biopsy by incubation with $0.25 \%$ trypsin-EDTA for 3 hours. Dissociated keratinocytes from the skin were seeded in a T25 flask with iЗT3 cells and grown in Green's medium ${ }^{17}$ supplemented with penicillin and streptomycin (100 unit / $100 \mu \mathrm{g}$ per mL, Pharmacy in GOSH, London, UK). After reaching confluence, cells were passaged and seeded in fresh flasks and transduced with lentiviral vector LV-INVO-SP. No antibiotics were added to Green's medium after this point ${ }^{17}$. Transduced cells were further cultured in $100 \mathrm{~mm}$ dishes to form epithelial sheets. Frozen irradiated murine fibroblasts $3 \mathrm{~T} 3$ cells (i3T3) were freshly thawed and added to keratinocyte cultures with a density of $0.6-1.6 \times 10^{6}$ cells / T25 flask. Epithelial sheets were harvested by $2 \mathrm{mg} / \mathrm{mL}$ dispase in PBS solution, then attached to Tricotex (Smith \& Nephew, Hull, UK), and packed in a sterile container for grafting. Frozen i3T3 cells and all media, reagents and chemistry for keratinocytes and sheet cultures were supplied by Regenerys (Sheffield, UK).

\section{H\&E staining, immunofluoresence staining and immunoblotting}

Punch skin biopsies taken from NS patients were formalin fixed and paraffin wax embedded, sectioned and stained with H\&E carried out at the Histopathology Department, GOSH NHS Foundation Trust. Immunofluorescence staining for LEKTI was performed on the paraffin sections ( $6 \mu \mathrm{m}$ thickness) using methods previously described ${ }^{15}$. A custommade affinity purified rabbit polyclonal antibody directed against the C-terminus of LEKTI ${ }^{15}$ was used (Eurogentec, Southamton, UK). A polyclonal anti- human KLK5 antibody (H-55, 
Santa Cruz, Heidelberg, Germany) was used at the dilution of 1:250 for the immunostaining of KLK5. Stained tissues were imaged using an upright light microscope (Zeiss Axiovert) or Zeiss confocal microscope LSM 710 (Zeiss, Cambridge, UK) and images were processed using Adobe Photoshop CS (Adobe Systems Inc, San Jose, Ca, USA). Immunoblotting for LEKTI expression was carried out in cells or sheet lysates using a monoclonal anti- LEKTI antibody (1C11G6, Invitrogen, Paisley, UK) as detailed previously ${ }^{15}$.

\section{Quantitative PCR}

Integrated vector copy number was assessed in transduced cells by quantitative PCR using primers (F-CAGGACTCGGCTTGCTGAAG and R-TCCCCCGCTTAATACTGACG) and probe (FAM-CGCACGGCAAGAGGCGAGG-TAMRA) designed to target the HIV Psi packaging element of the lentiviral vector LV-INVO-SP, and in comparison to signal generated for the housekeeping gene human albumin (F-GCTGCTATCTCTTGTGGGCTGT, RACTCATGGGAGCTGCTGGTTC, probe: VIC-CCTGTCATGCCCACACAAATCTCTCC-TAMRA). Integrated copy number was calculated with the aid of standard curves generated using plasmids encoding the vector and cDNA for human albumin.

\section{LAM-PCR and integration site mapping}

DNA from P003 was extracted from transduced and none-transduced epithelial sheets prior to grafting and a skin biopsy taken from the area of grafting at 3 months post grafting were used for lentiviral vector integration site analysis. LAM-PCR was used to identify lentiviral vector flanking genomic sequences in these samples as described previously ${ }^{18}$. Triplicates for each sample were amplified and subsequent library preparation ${ }^{19,20}$ was carried out for deep sequencing by MiSeq (Illumina) to determine lentiviral integration profiles and potential clonal dominance. Raw sequence data were trimmed according to sequence quality (Phred 30). Only sequences with $100 \%$ sequence identity barcodes were further analyzed. Primary IS annotation was conducted by using GENE-IS (version 1.2, Afzal et al, 2017). 
Image analysis

Immunofluorescence images were taken using a Zeiss confocal microscopy LSM710 and the intensities of optical sections were quantified using ImageJ. Briefly, three nonoverlapping but adjacent optical sections were recorded and saved digitally from each slide using a $40 \times$ NA1.2W objective. Acquisition settings were identical for all samples. Images were straightened by tracing a line along the cornified layer using ImageJ straighten function, then the fluorescence intensity was measured across a $150 \mu \mathrm{m}$ section to a depth of $250 \mu \mathrm{m}$. 3 images per sample were analyzed, and mean intensity plotted. Data was corrected for non-specific background staining from secondary antibody stained controls.

\section{Trans-epidermal water loss}

Trans-epidermal water loss (TEWL) was measured to assess skin barrier function before and after grafting used a VapoMeter (Delfin technologies, Surrey, UK). Three measurements were taken at each time point to obtain an average value.

\section{ELISPOT assay}

PBMCs were isolated from EDTA treated peripheral blood samples by density gradient centrifugation using Lymphoprep (Axis Shield, Oslo, Norway) and stored in liquid nitrogen. Immediately before ELISPOT assay, the cells were thawed and the number of viable cells was established using trypan blue staining. The human IFN- $\gamma$ ELISPOT Kit (BD Biosciences, Berkshire, UK) was used according to the manufacture instructions. Briefly, $1 \times 10^{5}$ of viable cells were seeded per well in a plate pre-coated with IFN- $\gamma$ antibody and incubated with $1.2 \mu \mathrm{g} / \mathrm{ml}$ full length rLEKTI (Oxford Expression Technologies, Oxford, UK) for 16 hours at $37^{\circ} \mathrm{C} .1 \mu \mathrm{g} / \mathrm{ml}$ Staphylococcal Enterotoxin B Fragment (Sigma, Dorset, UK) or 10\% FBS (Labtech International, Heathfield, UK) in RPMI medium (Life Technologies, Paisley, UK) were used as controls. Reaction plates were read using AID ELISPOT reader (AID GmbH, Strassberg, Germany) and positive spots quantified. 


\section{Statistics}

Statistical analysis of single factor ANOVA and post-hoc T test between two group were performed where applicable in the study data and a $p$ value of $\leq 0.05$ was used to determine statistical significance.

\section{Results}

\section{Subjects with Netherton syndrome}

Six patients (P001 - P006), two females and four males, aged 16-39 years, with Netherton syndrome were recruited to the study. Mutations in SPINK5 and baseline reduction of LEKTI expression were confirmed by Sanger sequencing in DNAs and immunostaining in skin biopsies (Supplementary information Table S1 and Figure S1). All patients were found to harbor compound heterozygous mutations in SPINK5 which caused frameshifts, premature termination of codons, or intron retention, resulting in truncated or abnormal LEKTI protein expression. In all cases skin swabs were taken prior to skin procurement for microbiological screening. Subjects with bacterial growth were treated with appropriate antibiotics for 10 days. Trans-epidermal water loss (TEWL) measurements, blood samples and photographs of the skin were collected for baseline recording.

\section{Generation of GMP compliant lentiviral gene modified epithelial sheets}

In compliance with regulatory and ethics approvals and with informed consent, a skin biopsy $(6 \mathrm{~mm})$ from the anterolateral aspect of the thigh was taken from each patient and primary keratinocytes, including keratinocyte stem cells, were isolated and co-cultured with irradiated 3T3 (i3T3) feeder cells in a T25 flask. Where skin resistant bacteria were detected on previous skin swabs, or if there was known hyper-sensitivity to penicillin, vancomycin and streptomycin (50 $\mathrm{g}$ or $100 \mu \mathrm{g}$ per $\mathrm{mL}$ ) were substituted in the culture. Keratinocytes were passaged when counts reached $\sim 3 \times 10^{6}$, and sub-cultured in three fresh T25 flasks at $1 \times 10^{6}$ cells per flask (P1), against with i3T3 cells. After 24 to 48 hours, P1 keratinocytes were exposed to a single round of lentiviral vector LV-INVO-SP $\left(2 \times 10^{8} \mathrm{IU}\right.$ $/ \mathrm{mL}$ ) transduction for 6 hours at a multiplicity of infection (MOI) of 20. One control flask 
was retained without transduction. P1 keratinocytes were then cultured and expanded to $\sim 2$ to $3 \times 10^{6}$ cells / flask, and passaged and re-seeded in a $100 \mathrm{~mm}$ culture dish for sheet culture (P2). Mature epithelial sheets in which cultured keratinocytes reached stable confluence formed in 2-3 weeks. One week prior to harvesting the sheet for grafting, one of two transduced sheets, and the non-transduced sheet, were harvested for assessments of transduction efficiency, including pro-viral copy number by qPCR, and LEKTI expression by in situ immunostaining and immunoblotting. Where release criteria were met ${ }^{17}$, the other transduced sheet (the Investigational Medicinal Product, IMP), was harvested following brief trypsinization of any residual i3T3 cells, and was then cultured in Green's medium (free of cholera-toxin) for three days. The cultured sheet was finally detached by Dispase treatment and subjected to final release checks, before delivery to the theatre for grafting (Supplementary information, Figure S2).

Five sheet cultures were generated from five individual skin biopsies from P002-P006. All cultured sheets were successfully transduced with LV-INVO-SP lentiviral vector with an average pro-viral copy number ranging from 1.8 -11.6 per cell (Supplementary information Table S2).

Successful transduction was further confirmed by detection of LEKTI expression using immunoblotting (Figure 1). A $\sim 148 \mathrm{kDa}$ full length LEKTI band was observed in samples from sheets generated using normal keratinocytes or transduced NS keratinocytes. There was an additional smaller band ( $120 \mathrm{kDa})$ in samples from normal keratinocytes/sheet but not in samples from transduced NS keratinocytes/sheets because the vectorized codon optimized SPINK5 CDNA encoded a longer 15 domain LEKTI isoform and not the alternatively spliced 13 domain LEKTI isoform ${ }^{21}$. Additional smaller bands were also detected in normal keratinocyte derived sheets and transduced NS sheets ${ }^{22}$. Neither full length nor cleaved LEKTI was detected in non-transduced NS samples.

All five patient-derived transduced keratinocyte populations progressed to epithelial sheet culture, with three out of five cultures (P002, P003 and P004) maturing sufficiently to form the requisite sheets over a 23 to 25 day period. These met release criteria based on scores for clarity, size, integrity, color and lift ability. There was comparable LEKTI expression in NS sheets transduced with LV-INVO-SP virus compared to the sheets generated using normal donor keratinocytes, whereas there was no LEKTI expression in 
control sheets generated using non-transduced NS keratinocytes, examined by in situ immunofluorescence staining (Figure 2). The total duration of the process from the expansion of newly isolated keratinocytes from a skin biopsy to sheet lifting was approximately 44 days.

Two epithelial sheets (P005 and P006) did not meet release criteria. These sheet cultures exhibited either overgrowth of fibroblasts or dominant differentiated keratinocytes in the epithelial cultures, which probably inhibited formation of a cohesive epithelial sheet (Supplementary information Figure S3). The overgrowth of fibroblasts was of human origin and not derived from murine 3 T3 feeder cells, as confirmed by qPCR using specific primers for murine SPINK5 DNA.

\section{Gene modified epithelial sheet grafting and clinical observations}

Two subjects with successfully generated grafts were withdrawn before grafting; one became unavailable within the scheduled grafting period, and the other was considered clinically unstable.

Patient P003 was prepared with a $4 \times 5 \mathrm{~cm}\left(20 \mathrm{~cm}^{2}\right)$ graft bed on the anterolateral aspect of the left thigh by a surgical de-epidermization under local anesthetic. The upper epidermis was removed and the gene modified epithelial sheet was attached on the deepidermized bed, with an externalized tricotex dressing. Mepitel soaked in saline was placed on the grafted area, and then Mepitel film was applied to secure the graft in place (Figure 3. b). A Coban ${ }^{\mathrm{TM}}$ dressing was further applied on top of Mepitel film. The patient was discharged from the hospital on the same day. Post-graft monitoring of the patient lasted 12 months. Visual inspection showed that the graft looked healthy with an epithelialized appearance within the first week, and complete healing with mild postinflammatory hyperpigmentation at one month (Figure $3 \mathbf{c}$ and $\mathbf{d}$ ). Three months post grafting, the graft had blended with the surrounding skin (Figure 3 e). There were no signs of infection or other complications.

\section{Transient detection of pro-viral vector and the expression of LEKTI in the graft}

Paired serial skin biopsies were taken at month 1, 3, 6 and 12 post grafting. Each pair included a biopsy from within the graft area and another one from outside the graft area 
$4 \mathrm{~cm}$ away from the edge of the graft. Pro-viral copy numbers, LEKTI expression and skin morphology were examined in the biopsies. Vector copy number (VCN) could be detected within the graft area after 3 months (0.0015 VCN/cell). The expression of LEKTI was also detected but at levels lower than in normal skin and declined over time until barely detectable by month 6 (Figure 4 . e - g). A very weak LEKTI signal was detected in the biopsy sample taken from outside the graft area at month 1 (Figure 4. i).

Lentiviral integration sites (ISs) were evaluated in non-transduced and transduced epithelial sheets prior to grafting and a skin biopsy taken from the area 3 months after grafting. LAM-PCR revealed a polyclonal integration site pattern for transduced epithelial sheet prior to grafting, but only revealed few bands in the skin biopsy sample, likely due to the limited amount of modified DNA recovered from the biopsy (Supplemental information, Figure S3a). Deep sequencing results obtained by MiSeq sequencing recovered 828,609 raw sequencing reads which revealed 215 unique and precisely mappable IS (Supplemental information, Table S3). Bioinformatic analyses of sequenced LAM-PCR amplicons found that transduced cells had a polyclonal integration site pattern with no preferred integration in or near genes previously involved in serious adverse events in other gene therapy studies (Supplemental information, Figure S3b).

Biopsy histology assessed by hematoxylin and eosin (H\&E) staining was comparable for hypergranulosis, psoriasiform cornification (integrity of cornified layer, parakeratosis, exfoliated corneocytes) and lympho-histiocytic infiltration ${ }^{17}$. Mild morphologic differences were noted including reduced rete ridges at month 1 and month 3 (Figure 5. c-f versus g-j) and a lower density of nuclei in the upper compartment of the epidermis within the graft area at months 1 and 3 (Figure 5. c-d) compared to the outside graft area at time points and late time points (Figure 5. g-h and e-f).

\section{Functional correction of LEKTI confirmed by expression patterns of kallikrein 5}

To evaluate functional restoration of LEKTI on gene modified sheets, the expression of KLK5, usually controlled by LEKTI, was examined. In the normal healthy skin, KLK5 was expressed and localized within the cornified layer of the epidermis, while in NS skin, KLK5 was displaced to the lower suprabasal layers of the epidermis (Figure 6. a and c). Image analysis captured these differences as a sharp intensity peak at the cornified layer in 
normal skin, compared to a shifted peak in suprabasal layers in P003 (Figure 6. $\mathbf{m}$ and $\mathbf{n}$ ). At month 1, the peak within the graft area was similar to that found in normal donor skin, indicating a correction of KLK5 expression (Figure 6. e and p). Interestingly, the area outside the grafted skin also showed a similar expression pattern to the normal skin (Figure 6, $\mathbf{f}$ and $\mathbf{q}$ ). However, KLK5 expression has reverted by months 3, 6 and 12 (Figure 6. $\mathbf{r}$ to $\mathbf{w})$. The ratio of KLK5 expression between cornified and suprabasal layers was significantly different in samples taken from normal and NS baseline skin, at months 1 and 3 outside skin samples, and at month 6 and 12 inside and outside skin samples $(p<0.05$, ANOVA and post-hoc T test). However there were no differences between normal and inside skin samples taken at month 1 and 3 ( $p=0.48$ and 0.06, ANOVA and post-hoc T test). All these findings were consistent with transient expression and functional correction of LEKTI within the gene modified epithelial graft.

\section{Skin barrier function and host immune responses to LEKTI}

Trans-epidermal water loss (TEWL) was also measured before and after grafting in the patient using a VapoMeter with results showing no significant changes of TEWL before and after grafting.

Immune response following sheet grafting was assessed by examining peripheral blood cellular interferon-y immune responses against the LEKTI protein using ELISPOT. There was an increased number of positive spots at month 12, which may have indicated cell mediated immunity against the graft, however, the difference compared to the baseline PBMC sample was not significant (ANOVA, $p=0.08$ ) (Table S4). Such assessments of systemic cellular responses may not reflect localized tissue immune activity, or capture possible antibody mediated responses, and secondary challenge was not part of the study design or approval.

\section{Discussion}

This study aimed to determine the feasibility and safety of lentiviral gene modified epithelial sheets in NS. Techniques for growing and expanding human keratinocytes including keratinocyte stem cells, and culture as an epithelial sheet for autografts has been 
applied for more than 25 years ${ }^{23}$ and are widely used in clinics for burn patients and chronic ulcers. This technique has been adopted in gene therapy for genetic skin conditions, in which autologous keratinocytes are genetically modified before being cultured as an epithelial sheet ${ }^{12}$. There is a recent successful case utilized a retroviral gene modified keratinocyte sheet therapy in junctional epidermolysis bullosa ${ }^{13}$. We used a similar approach successfully generating three gene modified epithelial sheets for autografting, demonstrating the feasibility of lentiviral gene modification of primary keratinocytes.

A self-inactivating lentiviral vector configuration was used as previous studies have reported that this vector system was safe for the modification of hematopoietic stem cells in conditions such as severe Wiskott-Aldrich syndrome, Adrenoleukodystrophy and Beta thalassemia ${ }^{24,25}$. However, vector integration analysis has rarely been performed on primary keratinocytes. A report for self-inactivating MLV vectors in primary keratinocytes previously reported a reduced 'genotoxic' profile in transduced cells ${ }^{26}$. Integration sites for lentiviral vectors in a spontaneously immortalised keratinocyte cell line (HaCat) were also reported with integration preferences for gene and gene-rich regions as expected ${ }^{27,28}$. In this first application of lentiviral modification of primary keratinocytes, we analysed integration sites in lentiviral gene modified epithelial sheets following lentiviral vector transduction and found no preference for integration sites in or near genes previously associated with serious adverse events such as MDS1, EVI1, LMO2 following gamma retroviral transduction of $\mathrm{HSC}^{29}$. The detection of multiple integration sites and polyclonal clonal contribution in primary keratinocytes suggested no clonal dominance had occurred during the ex-vivo culture period. Unfortunately the limited recovery of modified DNA from biopsy samples precluded detailed characterization of integration sites after grafting.

We found transient (less than 3 months) restoration of LEKTI expression and possible correction of a LEKTI down-stream protein KLK5 expression, which was consistent with 1-2 cycles of keratinocyte proliferation and differentiation. This in turn suggested that the epithelial sheet was derived mostly from paraclones/meroclones which exhibit relatively shorter replicative lifespans ${ }^{30}$. Skin derived keratinocytes are heterogeneous, containing differentiated keratinocytes and stem cells. Using the culture approach established by Rheinwald et al ${ }^{31}$, dissociated keratinocytes including keratinocyte stem cells can be 
cultured and form keratinocyte colonies in vitro. As only $0.1-1 \%$ of cells can form colonies which have a great influence on the expansion of the keratinocyte population, it is of vital important to ensure a high plating efficiency of freshly isolated keratinocytes to support colony formation. The most influential factor for plating efficiency was probably the density of irradiated $3 T 3$ feeder cells. $3 T 3$ feeder cells secrete soluble factors into the culture medium, and deposit basal lamina glycoproteins on the culture dish surface required for keratinocyte attachment, colony formation, prevention of early differentiation and avoidance of human fibroblast overgrowth ${ }^{32}$. Our pre-clinical data had indicated successful holo- clone formation and no evidence showed senescence and/or cytotoxicity in induced keratinocytes ${ }^{15}$ although pre-clinical study had used fresh irradiated 3T3 cells, whereas for the trial frozen $\mathrm{i} 3 \mathrm{~T} 3$ were used to ensure GMP compliance. Two previous reports of successful gene modified epidermal sheet therapy for JEB ${ }^{12,13}$ with the grafted epithelial sheet exhibiting functional gene expression for more than 6 years in the first case $^{33}$ and more than 3 years in the second case ${ }^{13}$. Their evidence suggested long-lasting, self-renewing keratinocyte stem cells had engrafted. Both trials used fresh made irradiated feeder cells. We used a commercially sourced supply of frozen i3T3 cells which had been previously characterized and banked with a certain level of batch to batch variation. The variation between batches of frozen i3T3 could further cause insufficient densities of feeder cells at the beginning of cultures, resulting in low plating efficiency and colony formation, overgrown fibroblasts and/or early differentiated keratinocytes. This could explain failure to form a cohesive epithelial sheet due to overgrowth of human fibroblasts and/or dominance of differentiated keratinocytes in two subjects. Thus, an additional inprocess quality controls in future studies may include clone forming assays and quantification of surrogate stem cell markers such as p63 and keratin 15 . Alternative readouts using single-cell RNA transcriptome analysis may allow identification of distinct stem cell populations within heterogeneous primary keratinocytes ${ }^{34}$. Approaches with feederfree keratinocyte culture system have also been developed but have yet to be fully evaluated in clinical trials ${ }^{14,35}$.

In the NS trial, it appears that residual basal layer cells may have persisted within the graft bed after a surgical de-epidermization, which manifested as differences in nuclei densities between upper and lower parts of the epidermis showed in Figure 1. c\&d. When 
cells dissociated from the shaved epidermis from the graft bed were cultured, almost no colonies grew in the culture (data not shown), suggesting that a proportion of original keratinocytes may have remained in the graft area and these cells subsequently competed with the grafted population. The depth of de-epidermization may be critical factor and bed preparation in NS may be more complicated than in JEB as NS patients retain intact dermaepidermal compartment. Surgical denuding of the area and complete elimination of the basal compartment, and its resident stem cells could result in scarring if the grafting process fails or is incomplete.

In conclusion, the lentiviral delivery platform and processes used for transducing primary keratinocytes and grafting of a modified autologous epithelial sheet were found safe and feasible. However, to ensure engraftment of long-lived stem cell populations and sustained protein expression, identification, isolation and modification of critical keratinocyte stem cell populations is required.

\section{Acknowledgments}

The helpful support of Fiona McKay, clinical genetics at GOSH, and Wang Wei at Genewerks, Germany. Supported by the Moulton Charity in the UK, Newlife for Disabled Children and the National Institute for Health Research (NIHR) Biomedical Research Centre based at Great Ormond Street Hospital/Institute of Child Health, and Guy's and St Thomas' NHS Foundation Trust and King's College London and the GSTT NIHR Clinical Research Facility. The views expressed are those of the author(s) and not necessarily those of the NHS, the NIHR or the Department of Health.

Dr Wei-Li Di is Great Ormond Street Hospital Children's Charity Senior Lecturer. Prof A Thrasher is a Wellcome Trust Senor Fellow in Clinical Science. Prof W Qasim is NIHR Research Professor (RP-2014-05-007)

\section{Conflict of interest}

The authors have declared that no competing financial interests exist in relation to this study. Profs Qasim and Thrasher hold interests in Orchard Therapeutics. 
References

1. Chavanas S, Bodemer C, Rochat A et al. Mutations in SPINK5, encoding a serine protease inhibitor, cause Netherton syndrome. NatGenet 2000;25:141-142.

2. Di WL, Harper J. Netherton Syndrome. In: Harper's Textbook of Pediatric Dermatology. Irvine AD, Hoeger PH and Yan AC, eds. (Blackwell Publishing Ltd, Hoboken, New Jersey, USA). 2011; pp. 124.121-124.110.

3. Magert HJ, Kreutzmann P, Drogemuller $\mathrm{K}$ et al. The 15-domain serine proteinase inhibitor LEKTI: biochemical properties, genomic organization, and pathophysiological role. EurJMedRes 2002;7:49-56.

4. Bitoun E, Micheloni A, Lamant L et al. LEKTI proteolytic processing in human primary keratinocytes, tissue distribution and defective expression in Netherton syndrome. HumMolGenet 2003;12:2417-2430.

5. Egelrud T, Brattsand M, Kreutzmann P et al. hK5 and hK7, two serine proteinases abundant in human skin, are inhibited by LEKTI domain 6. BrJDermatol 2005;153:12001203.

6. Bitoun $E$, Chavanas $S$, Irvine $A D$ et al. Netherton syndrome: disease expression and spectrum of SPINK5 mutations in 21 families. JInvest Dermatol 2002;118:352-361.

7. Ong C, O'Toole EA, Ghali L et al. LEKTI demonstrable by immunohistochemistry of the skin: a potential diagnostic skin test for Netherton syndrome. BrJDermatol 2004;151:1253-1257.

8. Bonnart C, Deraison C, Lacroix $\mathrm{M}$ et al. Elastase 2 is expressed in human and mouse epidermis and impairs skin barrier function in Netherton syndrome through filaggrin and lipid misprocessing. JClinlnvest 2010;120:871-882.

9. Borgono $\mathrm{CA}$, Michael IP, Komatsu $\mathrm{N}$ et al. A potential role for multiple tissue kallikrein serine proteases in epidermal desquamation. JBiolChem 2007;282:3640-3652.

10. Deraison C, Bonnart C, Lopez F et al. LEKTI fragments specifically inhibit KLK5, KLK7, and KLK14 and control desquamation through a pH-dependent interaction. MolBiolCell 2007;18:3607-3619.

11. Descargues P, Deraison C, Prost C et al. Corneodesmosomal cadherins are preferential targets of stratum corneum trypsin- and chymotrypsin-like hyperactivity in Netherton syndrome. JInvest Dermatol 2006;126:1622-1632. 
12. Mavilio F, Pellegrini G, Ferrari S et al. Correction of junctional epidermolysis bullosa by transplantation of genetically modified epidermal stem cells. NatMed 2006;12:13971402.

13. Hirsch $\mathrm{T}$, Rothoeft $\mathrm{T}$, Teig $\mathrm{N}$ et al. Regeneration of the entire human epidermis using transgenic stem cells. Nature 2017;551:327-332.

14. Siprashvili Z, Nguyen NT, Gorell ES et al. Safety and Wound Outcomes Following Genetically Corrected Autologous Epidermal Grafts in Patients With Recessive Dystrophic Epidermolysis Bullosa. JAMA 2016;316:1808-1817.

15. Di WL, Larcher F, Semenova E et al. Ex-vivo gene therapy restores LEKTI activity and corrects the architecture of Netherton syndrome-derived skin grafts. MolTher 2011;19:408-416.

16. Di WL, Semenova E, Larcher $F$ et al. Human Involucrin Promoter Mediates Repression-Resistant and Compartment-Specific LEKTI Expression. HumGene Ther 2012;23:83-90.

17. Di WL, Mellerio JE, Bernadis C et al. Phase I study protocol for ex-vivo lentiviral gene therapy for the inherited skin disease, Netherton Syndrome. HumGene TherClinDev 2013.

18. Schmidt M, Schwarzwaelder K, Bartholomae CC et al. Detection of retroviral integration sites by linear amplification-mediated PCR and tracking of individual integration clones in different samples. Methods in molecular biology (Clifton, NJ) 2009;506:363-372.

19. Gabriel R, Eckenberg R, Paruzynski A et al. Comprehensive genomic access to vector integration in clinical gene therapy. Nat Med 2009;15:1431-1436.

20. Paruzynski A, Arens A, Gabriel R et al. Genome-wide high-throughput integrome analyses by nrLAM-PCR and next-generation sequencing. Nature protocols 2010;5:13791395.

21. Tartaglia-Polcini A, Bonnart C, Micheloni A et al. SPINK5, the defective gene in netherton syndrome, encodes multiple LEKTI isoforms derived from alternative pre-mRNA processing. JInvest Dermatol 2006;126:315-324.

22. Fortugno $\mathrm{P}$, Bresciani $\mathrm{A}$, Paolini $\mathrm{C}$ et al. Proteolytic activation cascade of the Netherton syndrome-defective protein, LEKTI, in the epidermis: implications for skin homeostasis. JInvest Dermatol 2011;131:2223-2232. 
23. Munster AM. Cultured skin for massive burns. A prospective, controlled trial. Ann Surg 1996;224:372-375; discussion 375-377.

24. Booth C, Gaspar HB, Thrasher AJ. Treating Immunodeficiency through HSC Gene Therapy. Trends Mol Med 2016;22:317-327.

25. Hacein-Bey Abina S, Gaspar HB, Blondeau J et al. Outcomes following gene therapy in patients with severe Wiskott-Aldrich syndrome. JAMA 2015;313:1550-1563.

26. Cavazza A, Cocchiarella F, Bartholomae C et al. Self-inactivating MLV vectors have a reduced genotoxic profile in human epidermal keratinocytes. Gene therapy 2013;20:949957.

27. Almarza D, Bussadori G, Navarro $M$ et al. Risk assessment in skin gene therapy: viral-cellular fusion transcripts generated by proviral transcriptional read-through in keratinocytes transduced with self-inactivating lentiviral vectors. Gene therapy 2011;18:674-681.

28. Qian W, Wang Y, Li RF et al. Prolonged Integration Site Selection of a Lentiviral Vector in the Genome of Human Keratinocytes. Med Sci Monit 2017;23:1116-1122.

29. Ott MG, Schmidt $M$, Schwarzwaelder $K$ et al. Correction of X-linked chronic granulomatous disease by gene therapy, augmented by insertional activation of MDS1EVI1, PRDM16 or SETBP1. Nat Med 2006;12:401-409.

30. Barrandon $\mathrm{Y}$, Green $\mathrm{H}$. Three clonal types of keratinocyte with different capacities for multiplication. Proc Natl Acad Sci U S A 1987;84:2302-2306.

31. Rheinwald JG, Green H. Serial cultivation of strains of human epidermal keratinocytes: the formation of keratinizing colonies from single cells. Cell 1975;6:331-343.

32. Llames S, Garcia-Perez E, Meana A et al. Feeder Layer Cell Actions and Applications. Tissue Eng Part B Rev 2015;21:345-353.

33. De Rosa L, Carulli S, Cocchiarella F et al. Long-term stability and safety of transgenic cultured epidermal stem cells in gene therapy of junctional epidermolysis bullosa. Stem cell reports 2014;2:1-8.

34. Joost $S$, Zeisel $A$, Jacob $T$ et al. Single-Cell Transcriptomics Reveals that Differentiation and Spatial Signatures Shape Epidermal and Hair Follicle Heterogeneity. Cell systems 2016;3:221-237.e229. 
Page 20 of 35

20

35. Lenihan $C$, Rogers $C$, Metcalfe $A D$ et al. The effect of isolation and culture methods on epithelial stem cell populations and their progeny-toward an improved cell expansion protocol for clinical application. Cytotherapy 2014;16:1750-1759.

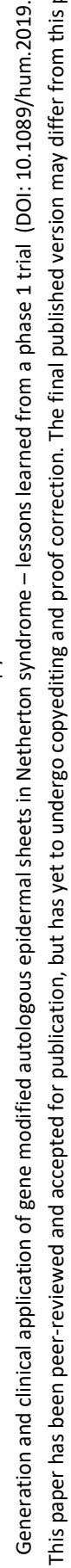


Figure Legends
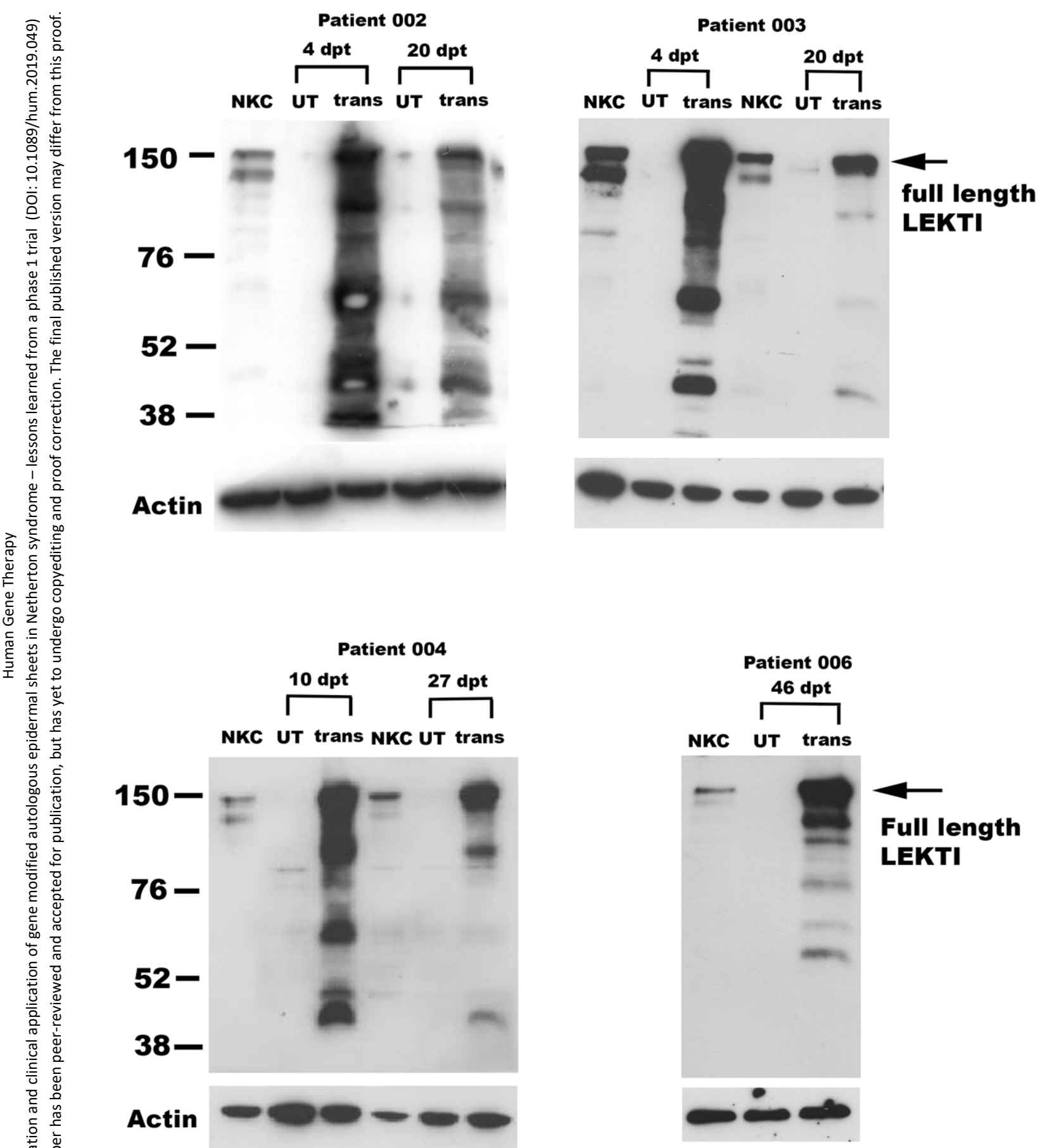

Figure 1. The expression of LEKTI in cultured keratinocytes and sheet transduced with the LV-INVO-SP virus. 
An over-expressed 15-domain LEKTI isoform band ( 148kDa) and several cleaved LEKTI with small sizes were detected in the transduced NS keratinocytes or sheets (trans) and normal keratinocytes and sheets (NKC). No positive bands were detected in nontransduced NS keratinocytes and sheets (UT). In each blotting, the first three lanes were loaded cell lysates from cultured keratinocytes prior to sheet culture. The last three lanes were loaded lysates from harvested sheet cultures, except for the patient 006 where only sheet samples were loaded. The samples from P005 were too little to perform the assay. The expression of $\beta$-actin was used as internal and loading control. $d p t=$ days post transduction. 


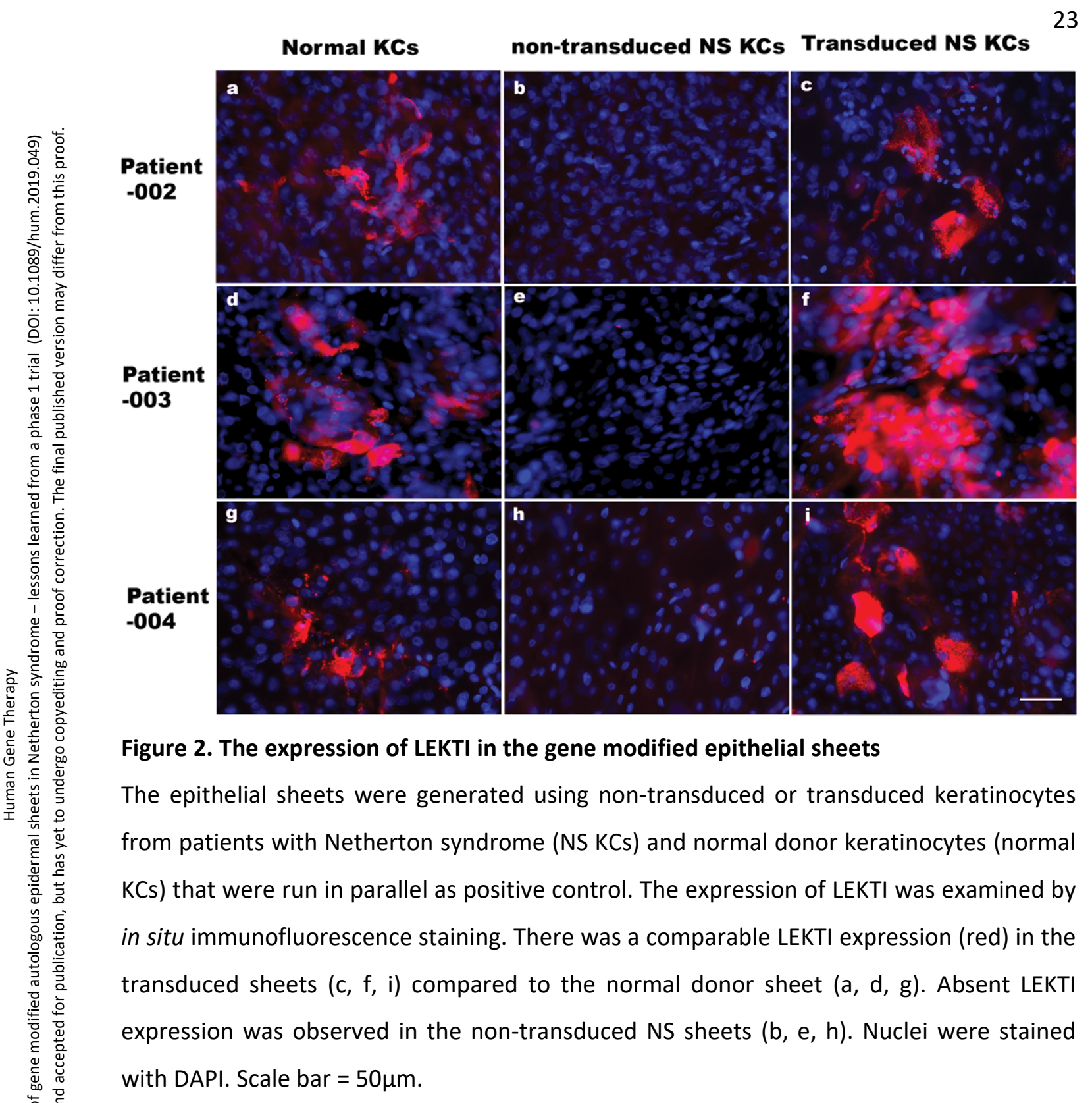




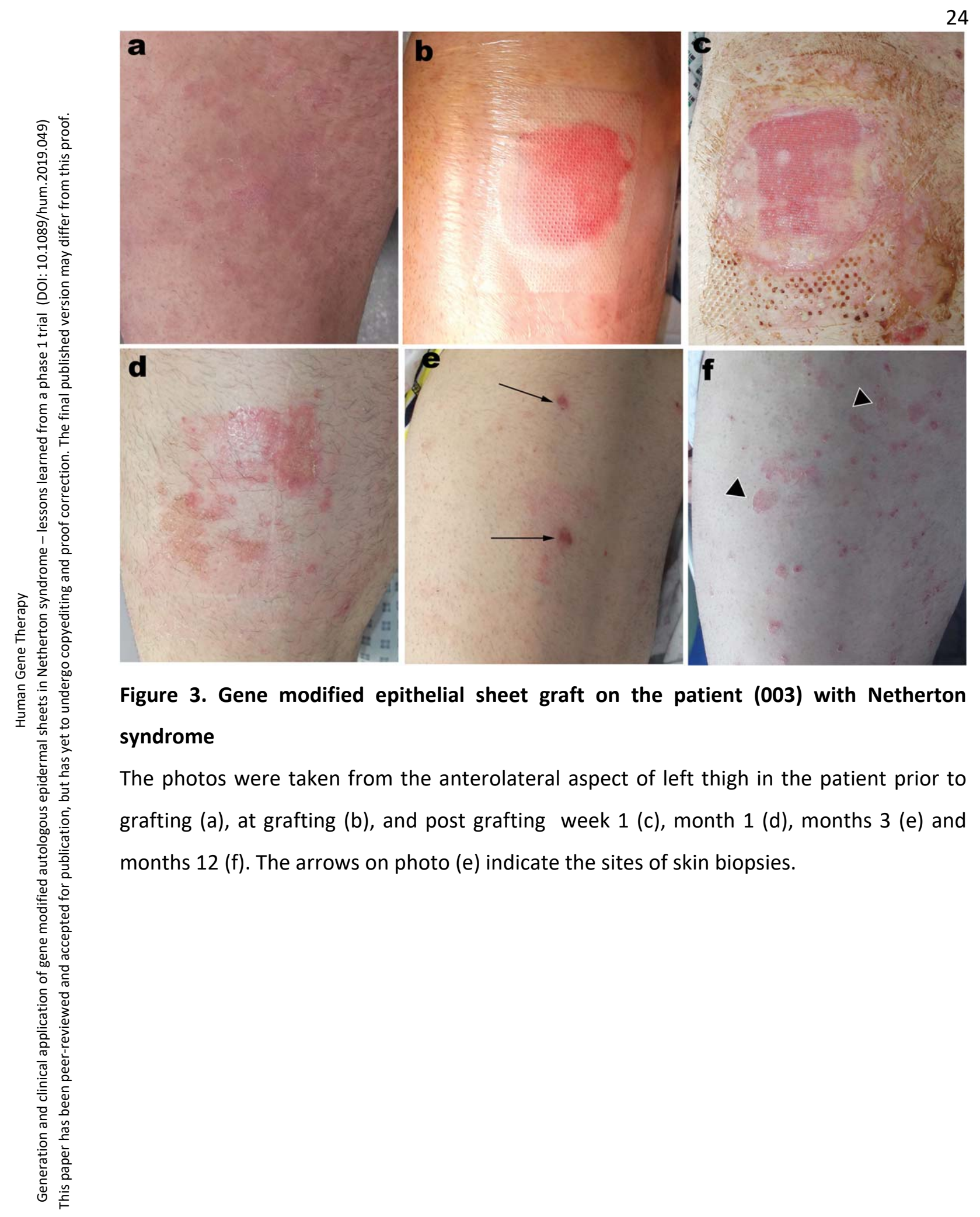




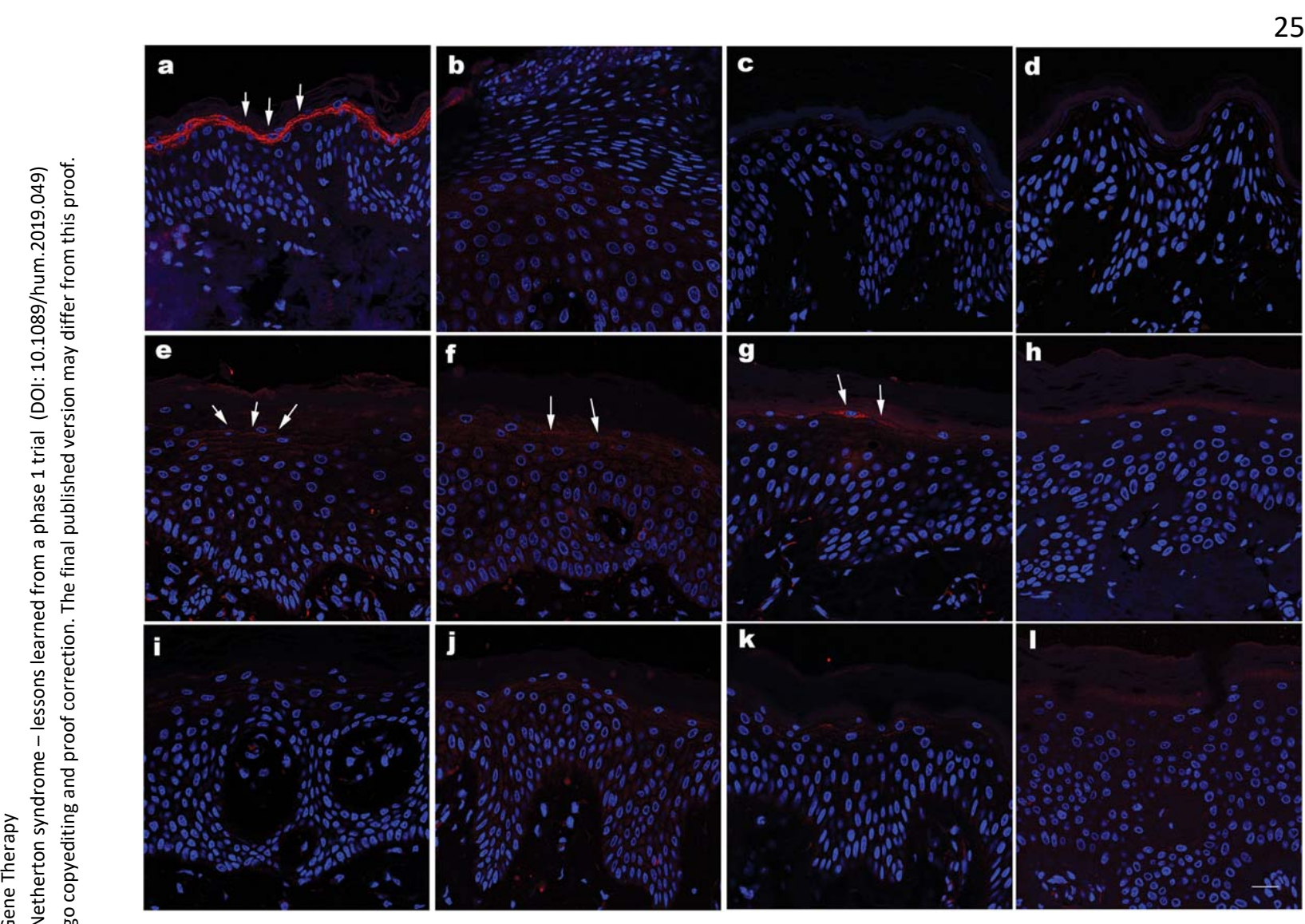

Figure 4. The expression of LEKTI in the grafted and outside grafted skin in P003

In the normal skin, LEKTI was highly expressed in the stratum granular layer (a, arrows), whereas, there was absent LEKTI expression in the baseline skin (b \& c). LEKTI expression was detected in the grafted skin at month 1 (e, arrows), month 3 (f, arrows) and month 6 (g, arrows) post grafting, but the levels were lower than in the normal skin. The LEKTI expression was barely detectable in month 12 (h). Very weak LEKTI expression was observed in the area of outside the grafted skin ( $i-k$, arrows). The staining without LEKTI antibody was used as negative control (d). Nuclei were stained with DAPI. Scale bar $=20$ $\mu \mathrm{m}$. 


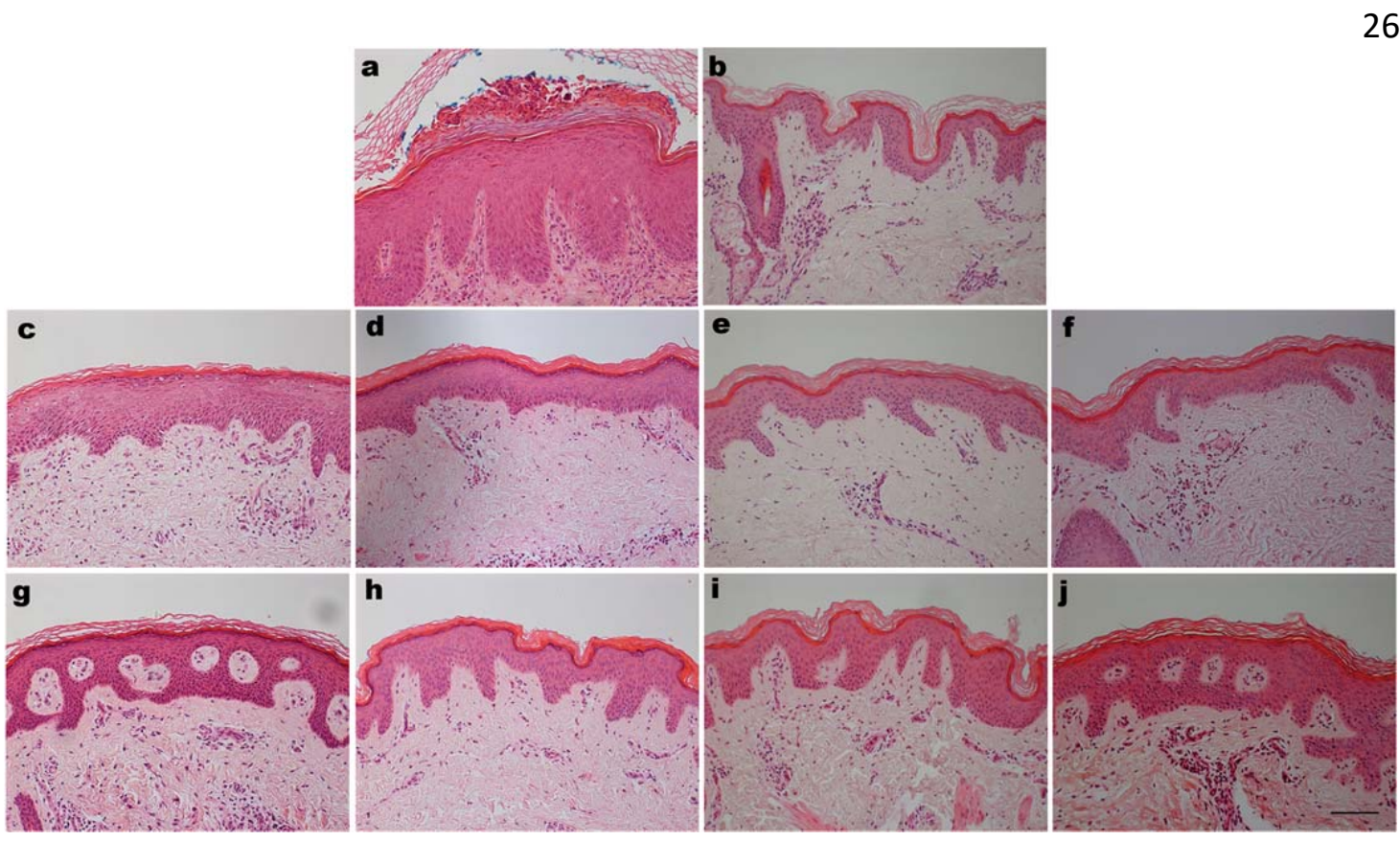

Figure 5. Histology of P003 skin within and outside the gene modified epithelial graft

Skin biopsies were taken from lesionnal skin (a) and non-lesional skin (b) before grafting, and the grafted skin at month 1 (c), months 3 (d), months 6 (e), months 12 (f), and outside the grafted skin at month 1 (g), months 3 (h), months 6 (i) and months 12 (j). Mild reduction in rete ridges were observed at month 1 (c) and months 3 on the grafted skin compared to the outside grafted skin (c, d versus $g, h$ ). A lower density of nuclei was observed in the top compartments of within the grafted skin at month 1 (c) and months 3 (d). Scale bar $=100 \mu \mathrm{m}$. 


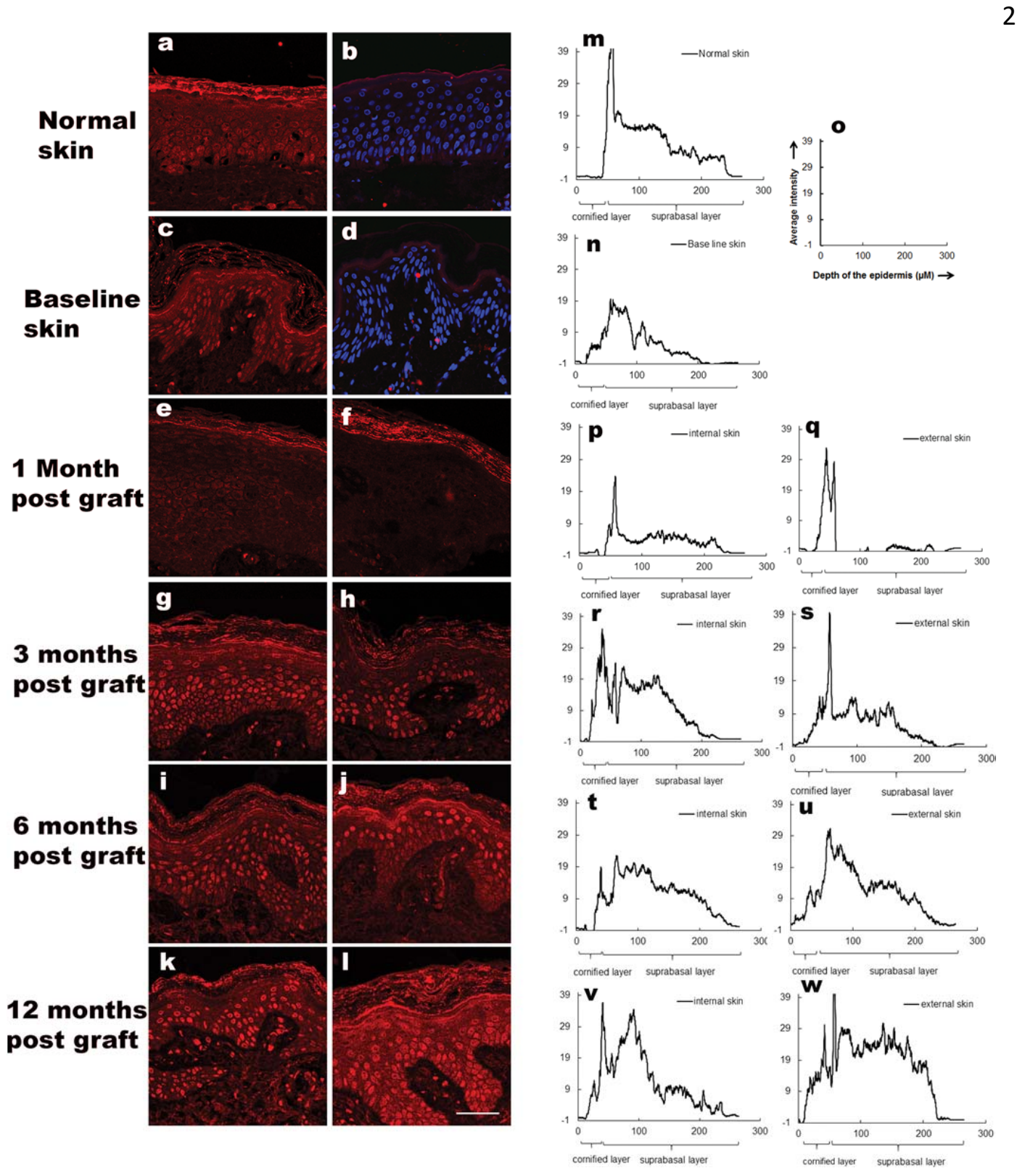

Figure 6. The expression of LEKTI down-stream target kallikrein 5 in the grafts

Reduced KLK5 (red colour) was observed in the grafted (e, g, i and k) and outside grafted ( $f$, h, j and I) skin at month $1(e, f)$ compared to NS baseline skin (c) but it was increased three months post grafting ( $g$ to I). The staining in normal skin (b) and baseline skin (d) without KLK5 antibody was used as negative control. Scale bar $=20 \mu \mathrm{m}$.

The $x$ and $y$ axis show the depth of the epidermis and fluorescence intensity respectively. $A$ sharp intensity peak located in the cornified layer was detected in the normal donor skin $(\mathrm{m})$, whereas a wide peak shifted to suprabasal layers was observed in the NS baseline skin (n). A corrected intensity profile was observed at month 1. 


\section{Supplementary Information}

\section{Supplementary Tables:}

\section{Table S1. Information for the six subjects recruited to the study}

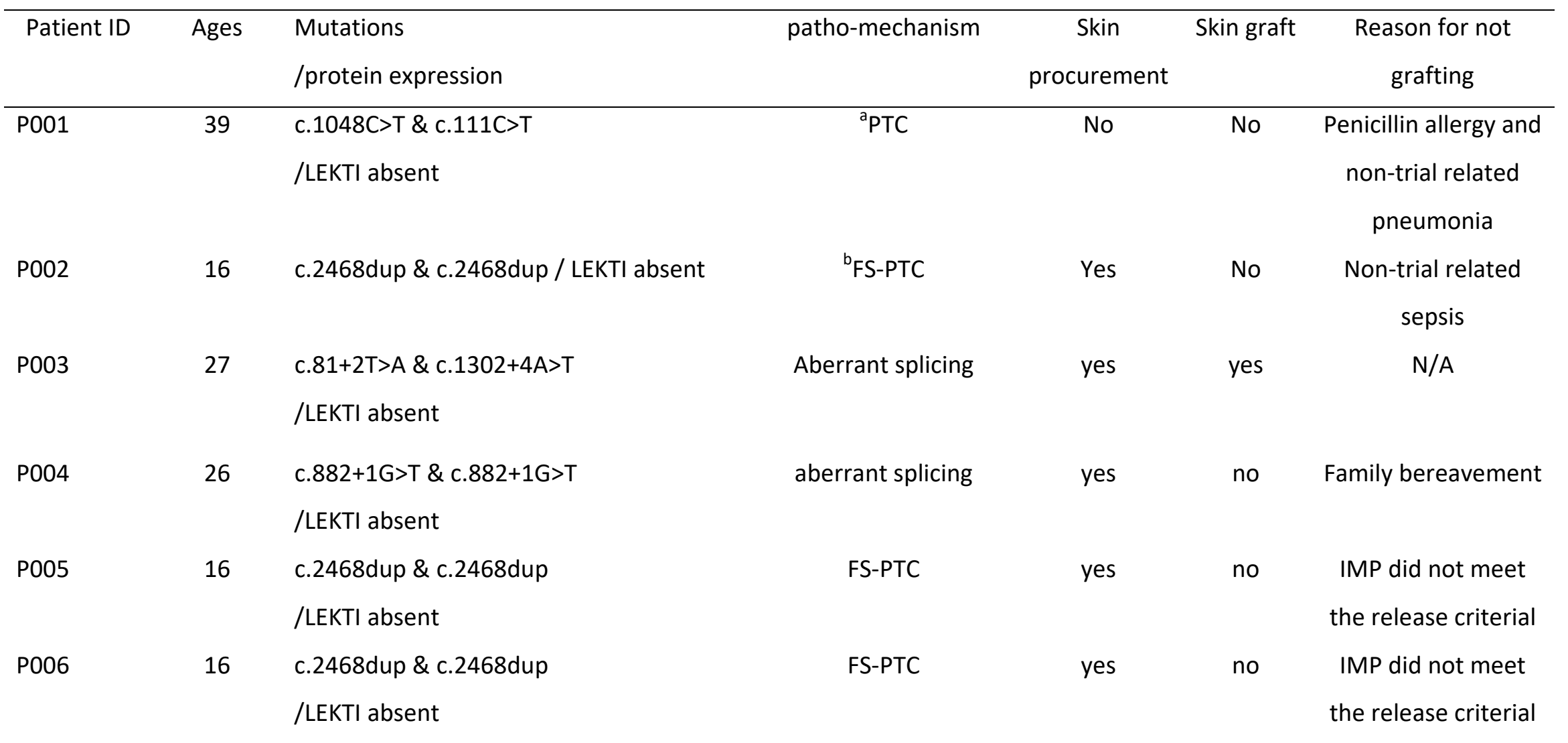

\footnotetext{
${ }^{a}$ premature termination codon; ${ }^{b}$ frame shift
} 
Table S2. Viral copy numbers assessed by qPCR

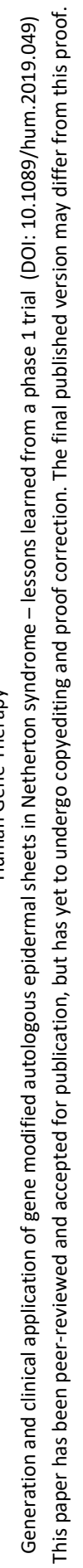

\begin{tabular}{ccc}
\hline patient & $\begin{array}{c}\text { Viral copy } \\
\text { numbers/cell }\end{array}$ & $\begin{array}{c}\text { Days } \\
\text { post transduction }\end{array}$ \\
\hline 002 & 1.8 & 20 days \\
003 & 2.2 & 20 days \\
004 & 6.6 & 27 days \\
005 & 7.2 & 19 days \\
006 & 11.6 & 40 days \\
mean \pm SD & $5.9 \pm 4.0$ & $25.2 \pm 8.9$ \\
\hline
\end{tabular}


Table S3. Deep sequencing results and IS retrieval

\begin{tabular}{|c|c|c|c|c|c|}
\hline Cell Type & Method & DNA[ng] & Enzyme & $\begin{array}{l}\text { Sequences } \\
\text { reads }\end{array}$ & Unique IS \\
\hline SkinBiopsyDNA & $\mathrm{LAM}^{\mathrm{a}}$ & 25 & $\mathrm{MluCl}$ & 0 & 0 \\
\hline SkinBiopsyDNA & LAM & 25 & $\mathrm{MluCl}$ & 0 & 0 \\
\hline SkinBiopsyDNA & LAM & 25 & $\mathrm{MluCl}$ & 0 & 0 \\
\hline TransducedCellDNA & LAM & 55 & $\mathrm{MluCl}$ & 106132 & 25 \\
\hline TransducedCellDNA & LAM & 55 & $\mathrm{MluCl}$ & 169361 & 25 \\
\hline TransducedCellDNA & LAM & 55 & $\mathrm{MluCl}$ & 132580 & 31 \\
\hline UntransducedDNA & LAM & 10 & $\mathrm{MluCl}$ & 0 & 0 \\
\hline UntransducedDNA & LAM & 10 & $\mathrm{MluCl}$ & 0 & 0 \\
\hline UntransducedDNA & LAM & 10 & $\mathrm{MluCl}$ & 0 & 0 \\
\hline SkinBiopsyDNA & LAM & 25 & Msel & 1 & 0 \\
\hline SkinBiopsyDNA & LAM & 25 & Msel & 0 & 0 \\
\hline SkinBiopsyDNA & LAM & 25 & Msel & 0 & 0 \\
\hline TransducedCellDNA & LAM & 55 & Msel & 153630 & 51 \\
\hline TransducedCellDNA & LAM & 55 & Msel & 139301 & 37 \\
\hline TransducedCellDNA & LAM & 55 & Msel & 127600 & 45 \\
\hline UntransducedDNA & LAM & 10 & Msel & 4 & 1 \\
\hline UntransducedDNA & LAM & 10 & Msel & 0 & 0 \\
\hline UntransducedDNA & LAM & 10 & Msel & 0 & 0 \\
\hline
\end{tabular}

${ }^{\mathrm{a}}$ Linear amplification-mediated PCR; 
Table S4. Results of ELISPOT assay

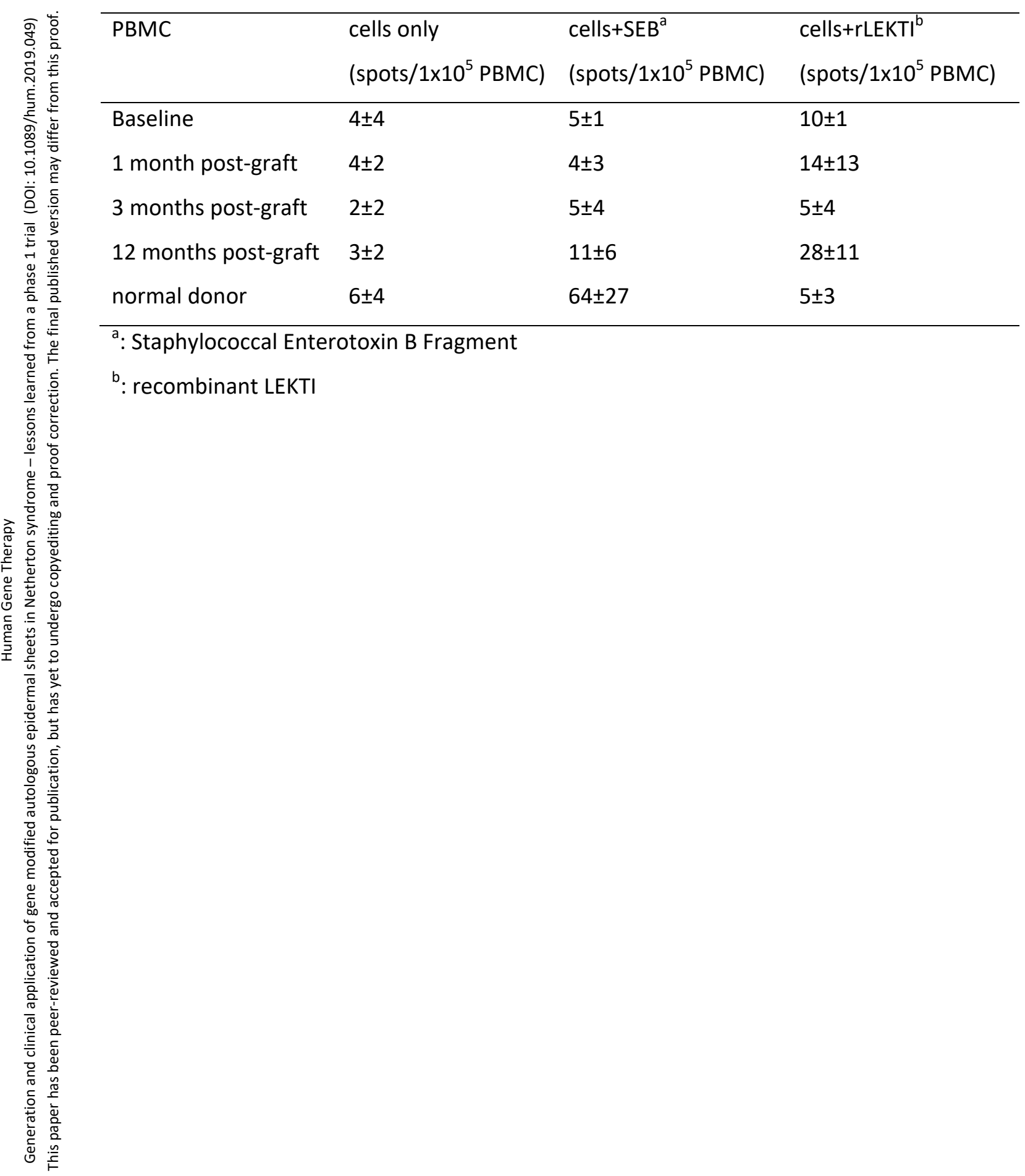


Supplementary Figures

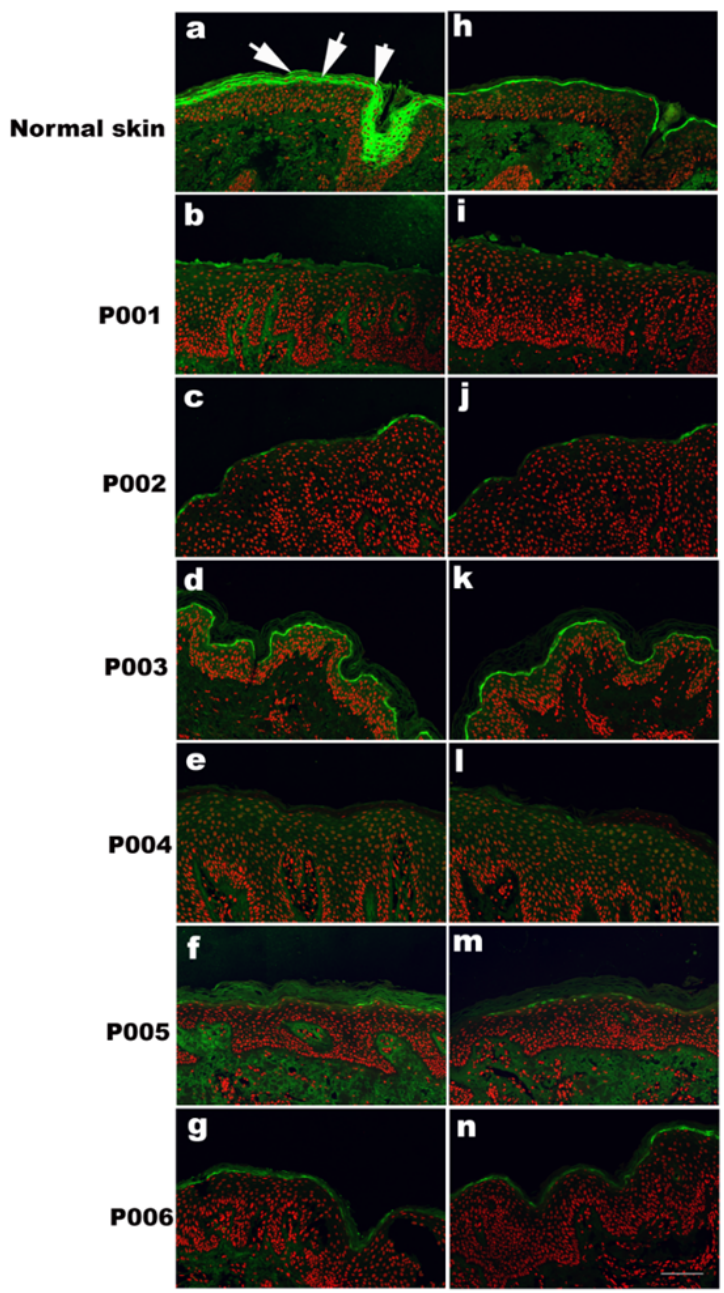

Figure S1. Absent expression of LEKTI in patients with Netherton syndrome

Immunofluorescence staining was performed on the paraffin sections of the skin from six patients with Netherton syndrome (NS) using an anti-human LEKTI antibody recognising an epitope at the c-terminus of the protein. The expression of LEKTI (green color, arrows) was detected in the stratum granular layer in the normal skin (a), wherease, absent LEKTI expression in the stratum granular layer was detected in all six NS patients ( $b$ to g). Since there was a non-specific staining of the secondary antibody in the stratum lucidum of the skin, the negative control staining with the secondary antibody alone was run in parallel to show the background staining for each sample (h-n). Scale bar $=100 \mu \mathrm{M}$. 


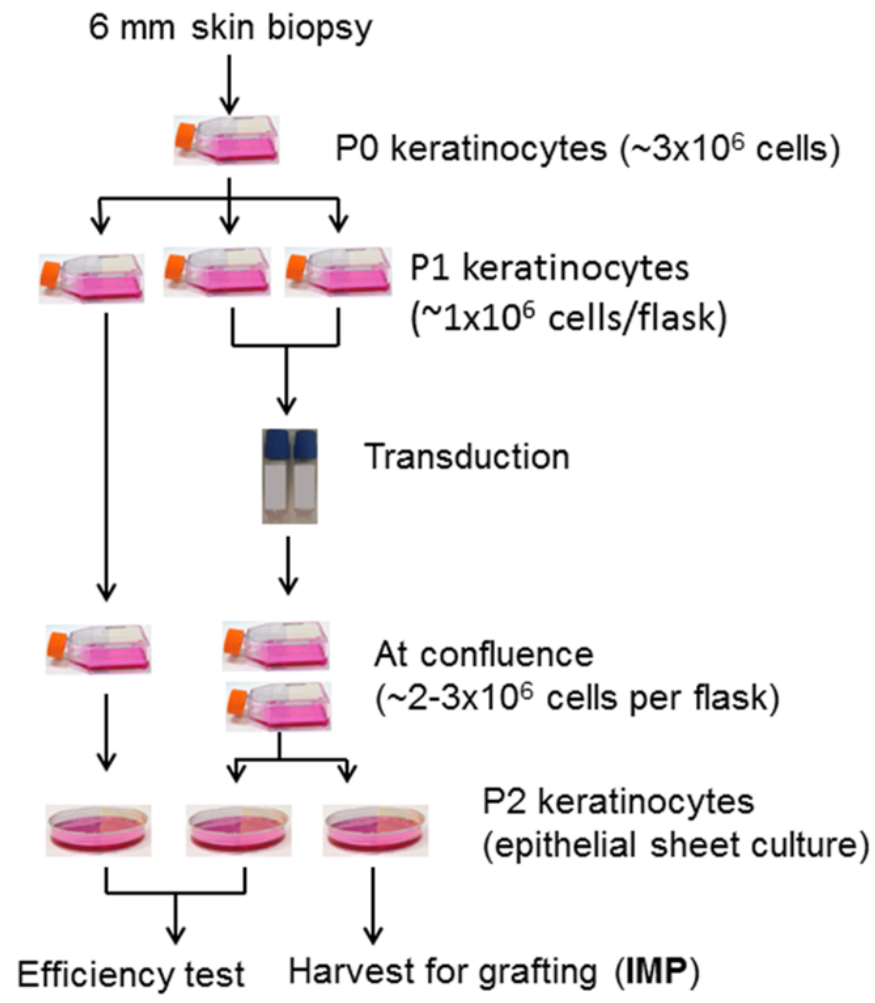

Figure S2. Flow chart for epithelial sheet culture

Schematic diagram showing the procedures of gene modified epithelial sheet culture. P0, $\mathrm{P} 1$ and $\mathrm{P} 2$ represent the passage numbers of keratinocytes. $\mathrm{IMP}=$ Investigational Medicinal Product. 

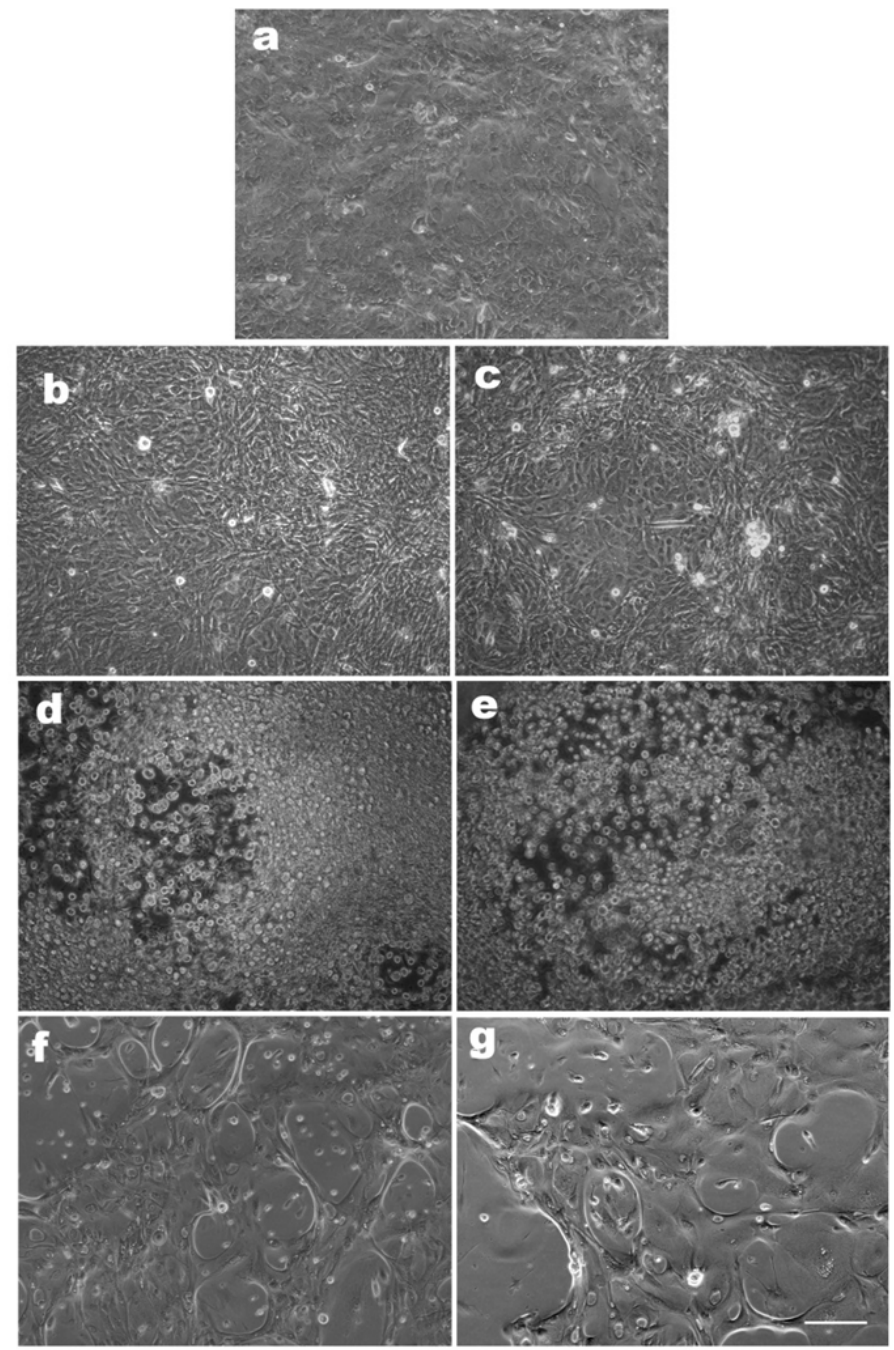

Figure S3. Epithelial sheet cultures which did not meet the release criteria

Epithelial sheet cultures were generated using normal keratinocytes (a) and keratinocytes from P005 (b-e) and P006 (f-g). Tightly packed small keratinocytes were observed in the sheet culture generated using normal donor keratinocytes. In contrast, in the P005 sheet culture, significant fibroblast growth was observed in both transduced (b) and nontransduced (c) cultures. This can be clearly demonstrated by brief trypsinization procedure, which removes the fibroblasts and leave gaps in the cultured sheet ( $d$ and $f$ ). In the P006 sheet culture, dominant large and irregularly shaped keratinocytes appeared in both transduced (f) and non-transduced (g) sheet cultures, suggesting the cultured keratinocytes were terminally differentiated. Scale bar $=200 \mu \mathrm{m}$. 
a

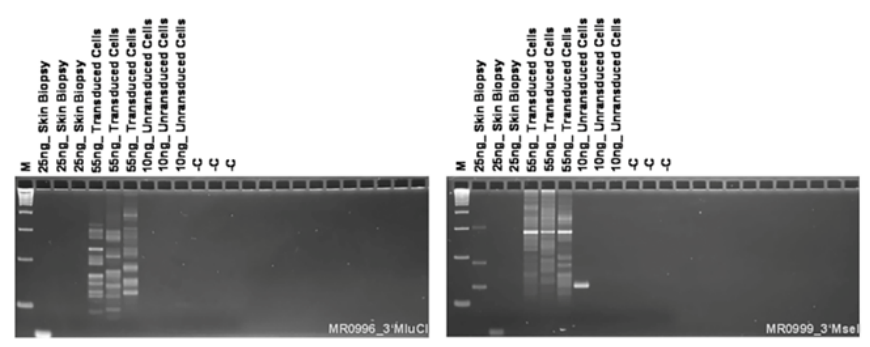

b

\begin{tabular}{lll}
\hline Rank & Transduced & Non-Transduced \\
\hline Top 1 & HDDC2 & EIF4G3 \\
Top 2 & CBARA1 & \\
Top 3 & UBR5 & \\
Top 4 & IPPK & \\
Top 5 & SGOL2 & \\
Top 6 & EDNRA & \\
Top 7 & KRT7 & \\
Top 8 & SPRY4 & \\
Top 9 & AJAP1 & \\
Top 10 & LYST & \\
\hline
\end{tabular}

Figure S4. LAM-PCR products and ten most prominent integration sites in transduced cells and skin from patient 003

The high-resolution electrophoresis gel image (a) shows LAM-PCR products from DNAs extracted from non-transduced and transduced epithelial sheet and the skin biopsy taken from the area of the grafting at 3 months post grafting from patient 003 . Multiple bands were observed in LAM-PCR products using the enzymes $\mathrm{MluCl}$ (left) and Msel (right). C = water and $\mathrm{M}=100 \mathrm{bp}$ ladder. Image (b) shows a heat map for integration sites. RefSeq gene name of 10 most prominent genes located next to (or at) the integration sites in transduced and non-transduced cells are listed in the table. There was no preferred integration in or near genes previously involved in serious adverse events in gene therapy. 